Article

\title{
Potential Infinity, Abstraction Principles and Arithmetic (Leśniewski Style)
}

\author{
Rafal Urbaniak ${ }^{1,2}$ \\ 1 Centre for Logic and Philosophy of Science, University of Ghent, 9000 Ghent, Belgium; \\ rfl.urbaniak@gmail.com \\ 2 Institute of Philosophy, Sociology and Journalism, University of Gdańsk, 80-309 Gdańsk, Poland \\ Academic Editor: Urszula Wybraniec-Skardowska \\ Received: 19 March 2016; Accepted: 2 June 2016; Published: 15 June 2016
}

\begin{abstract}
This paper starts with an explanation of how the logicist research program can be approached within the framework of Leśniewski's systems. One nice feature of the system is that Hume's Principle is derivable in it from an explicit definition of natural numbers. I generalize this result to show that all predicative abstraction principles corresponding to second-level relations, which are provably equivalence relations, are provable. However, the system fails, despite being much neater than the construction of Principia Mathematica (PM). One of the key reasons is that, just as in the case of the system of PM, without the assumption that infinitely many objects exist, (renderings of) most of the standard axioms of Peano Arithmetic are not derivable in the system. I prove that introducing modal quantifiers meant to capture the intuitions behind potential infinity results in the (renderings of) axioms of Peano Arithmetic (PA) being valid in all relational models (i.e. Kripke-style models, to be defined later on) of the extended language. The second, historical part of the paper contains a user-friendly description of Leśniewski's own arithmetic and a brief investigation into its properties.
\end{abstract}

Keywords: arithmetic; potential infinity; finite models; Leśniewski's systems; Leśniewski's Ontology; neologicism; abstraction principles

\section{Leśniewski's Ontology}

Since I will be looking at arithmetic whose underlying system is Leśniewski's higher-order logic called Ontology — whose features (syntactic flexibility and the fact that it is a free logic) make it particularly fit for the project-I will start with explaining what this system looks like. Readers familiar with the system can safely skip the section. Many examples of the general notions introduced in this section are available in further sections.

Ontology is built over Protothetic which is concerned with propositional aspects of reasoning. Since the propositional moves in what follows are not too complicated, the latter can be safely ignored for our current purposes. (For more details about all Leśniewski's systems, see [1].)

The construction of the language of the system starts with introducing a category of names $(n)$, to which belong name variables $a, b, c, \ldots$, which can be bound by quantifiers, and the predication copula: $\varepsilon$ used to build expressions of the category of sentences (s), which can be used to build other formulas by means of Boolean connectives. Ontology differs from the classical predicate logic in a few respects:

- There is no distinction between names and predicates. There is only one category of names, no matter whether they are empty, singular or common terms. Thus, a wide understanding of the word name is accepted here. Some examples of names in this wide understanding are: a cat, Socrates, brown, the bald guy in the corner, unicorn. 
- The language also contains variables of higher categories, insofar as those categories can be "built" by combining categories of names and of sentences (more about this soon).

- Predication is represented differently, by means of a predication copula $\varepsilon$. An expression of the form $a \varepsilon b$ is read as ' $a$ is $b^{\prime}$ (or ' $a$ is one of $b^{\prime} \mathrm{s}^{\prime}$ ), and it is true (roughly) if and only if $a$ is a singular term that names an object that is among the objects named by $b$. Thus, the following are true: Socrates \& philosopher, The book on my desk $\varepsilon$ Kleene's "Introduction to Metamathematics", and the following are well-formed, but false: 'Unicorn $\varepsilon$ animal', 'elephant $\varepsilon$ animal' (both are false because the subject fails to refer to exactly one object).

- Existential quantifier does not commit to objects. Existence is defined by $\forall a(e x(a) \equiv \exists b b \varepsilon a)$, and it is provable in Ontology that, for some $a$, $a$ does not exist (or, in a more meta-linguistic reading, that there is an empty name): $\exists a \neg \operatorname{ex}(a)$.

Besides name variables, Boolean connectives, brackets, and quantifiers binding name variables, expressions of categories that can be "built" from s's (the category of sentences) and n's are allowed. All (atomic) formulas of type $a \varepsilon b$ are of category s. Name variables are of category $n$. Any expression with which $k$ arguments of categories $\sigma_{1}, \ldots, \sigma_{k}$ yields an expression of category $\sigma$ is of category $\frac{\sigma}{\sigma_{1} \cdots \sigma_{k}}$. For instance, $\varepsilon$ takes two arguments, both of category $n$, and forms an expression of category $s$. Thus, its category is $\frac{s}{n n}$. Boolean combinations of expressions of category $s$ also belong to the language and are of category $s$.

For any semantic category of Ontology, constants (possibly with parameters) can be defined, and variables together with quantifiers binding those variables can be introduced. For any defined constant (possibly with parameters) of category $\frac{\tau}{\sigma_{1} \cdots \sigma_{n}}$ (rules of definitions are given below), (Leśniewski did not have a specified list of constants. Rather, he invented symbols ad hoc as he went.) There are also variables of that category, $f^{\frac{\tau}{\sigma_{1} \cdots \sigma_{n}}}, g^{\frac{\tau}{\sigma_{1} \cdots \sigma_{n}}}$, possibly with numerical subscripts. In practice, the superscripts are omitted when the semantic category is obvious from the context. (This is not Leśniewski's original device. His main tool was introducing different shapes of brackets surrounding the arguments.) If $\phi$ is of category $\frac{\tau}{\sigma_{1} \cdots \sigma_{n}}$ and expressions $\alpha_{1}, \ldots, \alpha_{n}$ are of categories $\sigma_{1}, \ldots, \sigma_{n}$, then $\phi\left(\alpha_{1}, \ldots, \alpha_{n}\right)$ is a well-formed expression of the language of Ontology of category $\tau$. If $\phi$ is an expression of category $s$ and $\zeta$ a variable of any category, then $\forall \zeta \phi$ and $\exists \zeta \phi$ is a well-formed formula of category $s$.

Ontology [2,3], in its version from 1920 has a single specific axiom:

$$
\forall a, b[a \varepsilon b \equiv \exists c(c \varepsilon a) \wedge \forall c, d(c \varepsilon a \wedge d \varepsilon a \rightarrow c \varepsilon d) \wedge \forall c(c \varepsilon a \rightarrow c \varepsilon b)] .
$$

This axiom says that, for any $a$ and $b, a$ is $b$ iff (i) for some $c, c$ is $a$; (ii) for any $c$ and $d$, if $c$ is $a$ and $d$ is $a$, then $c$ is $d$; and (iii) for any $c$, if $c$ is $a$, then $c$ is also $b$. The intended interpretation of $\varepsilon$ is that $a \varepsilon b$ is true if and only if there is exactly one object which is $a$, and this object is among the objects which are $b$ (this includes the case where $b$ names exactly one object). (This interpretation is not determined by the axiomatic basis of Ontology (see [4]).) Accordingly, (i) is supposed to guarantee the non-emptiness of $a$; (ii) is intended to express the requirement that $a$ names at most one object; and (iii) is meant to state the inclusion between $a$ and $b$ ('any object which is $a$, is $b^{\prime}$ ).

In the simplified version (equivalent to the original) of the system, we can use standard rules for quantifiers and apply propositional logic just as is it done in predicate logic. Quantifier elimination and introduction for variables of other categories are just an extension of classical rules to variables of higher categories. In addition, there are a few specific rules, especially connected with definitions and introduction of variables of different semantic categories. Definitions in Leśniewski's systems were creative, mainly because the system did not contain comprehension axioms and every higher-order existential claim was to be proven by defining an appropriate witness and proving that it has the desired property. (For more details on Leśniewski and definitions, see [5].) 
Any definition of a constant name expression of category $n$ (possibly with parameters $\zeta_{1}, \ldots, \zeta_{k}$ ) has the form:

$$
\forall a\left[a \varepsilon \Delta_{\zeta_{1}, \ldots, \zeta_{k}} \equiv a \varepsilon a \wedge \phi\left(\zeta_{1}, \ldots, \zeta_{k}\right)\right]
$$

where $\Delta_{\zeta_{1}, \ldots, \zeta_{k}}$ is the defined term, and $\phi$ is a formula with $a$ and $\zeta_{1}, \ldots, \zeta_{k}$ as its only free variables.

Protothetical definitions: for any variables $\alpha_{1}, \ldots, \alpha_{k}$ of semantic categories $\sigma_{1}, \ldots, \sigma_{k}$ and any formula $\beta$ with $\alpha_{1}, \ldots, \alpha_{k}$ (and possibly $\zeta_{1}, \ldots, \zeta_{u}$ ) as its only free variables, there are constants $\tau, \tau^{\prime}$ of categories $\frac{s}{\sigma_{1} \cdots \sigma_{k}}$ and $\frac{n}{\sigma_{1} \cdots \sigma_{k}}$ (possibly with parameters $\zeta_{1}, \ldots, \zeta_{u}$ ), the former introduced by the universal closure of (1), the latter introduced by the universal closure of (2):

$$
\begin{array}{r}
\tau_{\zeta_{1}, \ldots, \zeta_{u}}\left(\alpha_{1}, \ldots, \alpha_{k}\right) \equiv \beta, \\
a \varepsilon \tau_{\zeta_{1}, \ldots, \zeta_{u}}^{\prime}\left(\alpha_{1}, \ldots, \alpha_{k}\right) \equiv a \varepsilon a \wedge \beta .
\end{array}
$$

Now, the rules of so-called many-linked definitions (think of them as definitions with parameters). Suppose:

$$
\begin{array}{ccccc}
\chi_{1}^{\sigma_{1}} & \ldots & \chi_{k}^{\sigma_{1}} & \text { are variables of category } & \sigma_{1} \\
\chi_{1}^{\sigma_{2}} & \ldots & \chi_{l}^{\sigma_{2}} & \text { are variables of category } & \sigma_{2} \\
\vdots & \vdots & \vdots & \vdots & \vdots \\
\chi_{1}^{\sigma_{m}} & \ldots & \chi_{n}^{\sigma_{m}} & \text { are variables of category } & \sigma_{m}
\end{array}
$$

and $\beta$ is a formula with the above listed variables (and possibly $\zeta_{1}, \ldots, \zeta_{u}$ ) as its only free variables. Then, there is a constant $v$ (or possibly $v_{\zeta_{1}, \ldots, \zeta_{u}}$ ) of category:

$$
\frac{s}{\underbrace{\sigma_{1}, \ldots, \sigma_{1}}_{k}, \underbrace{\sigma_{2}, \ldots, \sigma_{2}}_{l}, \ldots, \underbrace{\sigma_{m}, \ldots, \sigma_{m}}_{n}}
$$

defined by the universal closure of:

$$
v_{\zeta_{1}, \ldots, \zeta_{u}}\left(\chi_{1}^{\sigma_{1}}, \ldots, \chi_{k}^{\sigma_{1}}\right)\left(\chi_{1}^{\sigma_{2}}, \ldots, \chi_{l}^{\sigma_{2}}\right) \cdots\left(\chi_{1}^{\sigma_{m}}, \ldots, \chi_{n}^{\sigma_{m}}\right) \equiv \beta
$$

where the lower index next to $v$ is optional.

Finally, the (two-fold) rule of extensionality is:

- If $\alpha$ and $\beta$ are nominal variables and $\gamma$ is a nominal variable distinct from them both, any formula of the form:

$$
\forall \gamma[\gamma \varepsilon \alpha \equiv \gamma \varepsilon \beta] \rightarrow \forall \chi[\chi(\alpha) \equiv \chi(\beta)]
$$

is a theorem (where $\chi$ is a variable of category $\frac{s}{n}$ ).

- Further, suppose:

- $\tau^{1}$ and $\tau^{2}$ are constants of category $\sigma=\frac{s}{\sigma_{1} \cdots \sigma_{k}}$ (possibly with parameters $\zeta_{1}, \ldots, \zeta_{u}$ ),

- $\tau^{1^{\prime}}$ and $\tau^{2^{\prime}}$ are constants of category $\sigma^{\prime}=\frac{n}{\sigma_{1} \cdots \sigma_{k}}$ (possibly with parameters $\zeta_{1}, \ldots, \zeta_{u}$ ),

- $\alpha_{1}, \ldots, \alpha_{k}$ are distinct variables of categories $\sigma_{1}, \ldots, \sigma_{k}$,

- $\zeta_{s}$ is a variable of category $\frac{s}{\sigma}$,

- $\zeta_{n}$ is a variable of category $\frac{s}{\sigma^{\prime}}$. 
Then, all instances of the following two schemata are theorems:

$$
\begin{aligned}
\forall \zeta_{1}, \ldots, \zeta_{u}, \alpha_{1}, \ldots, \alpha_{k}\left[\tau_{\zeta_{1}, \ldots, \zeta_{u}}^{1}\left(\alpha_{1}, \ldots, \alpha_{k}\right)\right. & \left.\equiv \tau_{\zeta_{1}, \ldots, \zeta_{u}}^{2}\left(\alpha_{1}, \ldots, \alpha_{k}\right)\right] \rightarrow \\
\rightarrow \forall \zeta_{s}, \zeta_{1}, \ldots, \zeta_{u}\left[\zeta_{s}\left(\tau_{\zeta_{1}, \ldots, \zeta_{u}}^{1}\right)\right. & \left.\equiv \zeta_{s}\left(\tau_{\zeta_{1}, \ldots, \zeta_{u}}^{2}\right)\right], \\
\forall \zeta_{1}, \ldots, \zeta_{u}, \alpha_{1}, \ldots, \alpha_{k}\left[a \varepsilon \tau_{\zeta_{1}, \ldots, \zeta_{u}}^{1^{\prime}}\left(\alpha_{1}, \ldots, \alpha_{k}\right)\right. & \left.\equiv a \varepsilon \tau_{\zeta_{1}^{\prime}, \ldots, \zeta_{u}}^{2^{\prime}}\left(\alpha_{1}, \ldots, \alpha_{k}\right)\right] \rightarrow \\
\rightarrow \forall \zeta_{n}, \zeta_{1}, \ldots, \zeta_{u}\left[\zeta_{n}\left(\tau_{\zeta_{1}, \ldots, \zeta_{u}}^{1^{\prime}}\right)\right. & \left.\equiv \zeta_{n}\left(\tau_{\zeta_{1}, \ldots, \zeta_{u}}^{2^{\prime}}\right)\right] .
\end{aligned}
$$

Roughly speaking, the rule of extensionality says that if two names refer to the same things, or two functors apply to the same arguments, then whatever is true of one of them is also true of the other.

Before we move on, a notational remark. The system, officially, has only one sort of variables of category $n$-these are lower-case letters from the beginning of the alphabet, possibly with numerical subscripts. However, Leśniewski and Leśniewskian scholars sometimes use the convention that, in the context where it is clear that a formula can be satisfied only under an interpretation that interprets a given variable as if it was singular, a capital letter is used. We will follow this convention, but keep in mind that technically nothing would change if all such capital letters were replaced with lower-case name variables.

\section{Arithmetic within Ontology}

Leśniewski's own approach to arithmetic was in disagreement with his nominalism. He simply gave a rather unsurprising axiomatization of numbers as objects.

The interesting question, however, is whether a more foundational approach can be taken by means of the logical devices developed by Leśniewski: how much of arithmetic can be constructed within Ontology? It turns out that quite a lot can be done. Note that, at this stage, the specific features of Ontology (it being a free logic and the way definitions in Ontology work especially) do matter, and so we move back to the official system. Let us see Ontology in action.

First, let us start with some basic definitions:

$$
\begin{array}{r}
\forall A[A \varepsilon \Lambda \leftrightarrow A \varepsilon A \wedge \neg A \varepsilon A], \\
\forall A[A \varepsilon V \leftrightarrow A \varepsilon A], \\
\forall a[E(a) \leftrightarrow \exists A A \varepsilon a], \\
\forall a[N(a) \leftrightarrow \neg \exists A A \varepsilon a], \\
\forall a[!(a) \leftrightarrow \forall B, C(B \varepsilon a \wedge C \varepsilon a \rightarrow B \varepsilon C)], \\
\forall a[E !(a) \leftrightarrow E(A) \wedge !(A)], \\
\forall a, b[\forall A(a \subset b \leftrightarrow A \varepsilon a \rightarrow A \varepsilon b)], \\
\forall a, b[a \mid b \leftrightarrow \neg \exists A(A \varepsilon a \wedge A \varepsilon b)], \\
\forall a, b[a \equiv b \leftrightarrow \forall A(A \varepsilon a \leftrightarrow A \varepsilon b)], \\
\forall A, B[A=B \leftrightarrow A \varepsilon B \wedge B \varepsilon A], \\
\forall A, a, b[A \varepsilon a \cup b \leftrightarrow A \varepsilon a \vee A \varepsilon b], \\
\forall A, a, b[A \varepsilon a-b \leftrightarrow A \varepsilon a \wedge \neg A \varepsilon b], \\
\forall A, a, b[A \varepsilon a \oplus b \leftrightarrow a \mid b \wedge(A \varepsilon a \cup b)], \\
\forall f, g[f \approx g \leftrightarrow \forall a(f(a) \leftrightarrow g(a))] .
\end{array}
$$

Definition (3) introduces a new constant empty term of category $n$. The right-hand condition is contradictory, and so nothing is $\Lambda$. Similarly, Definition (4) introduces a new constant universe of category $n$. Any individual satisfies the right-hand condition, and so $V$ applies to all objects in the domain (if there are any). 
Definitions (5)-(8) are definitions of expressions of category $s / n$-predicates which apply to names. $E(a)$ says that $a$ is empty, $N(a)$ says that $a$ is non-empty, !( $a)$ says that at most one object is $a$, and $E !(a)$ says that exactly one object is $a$.

Definitions (9)-(12) introduce new constant symbols of category $s / n, n . a \subset b$ says that all $a$ are $b$ s, $a \mid b$ says that no $a$ is $b, a \equiv b$ says that $a$ s and $b$ s are exactly the same objects, and $A=B$ says that $A$ and $B$ is one and the same object.

Definitions (13)-(15) introduce new constants of category $n / n, n$. $a \cup b$ s are objects that are either $a$ s or $b \mathrm{~s}, a-b$ are objects which are $a$ s but not $b \mathrm{~s}$, and $a \oplus b$ s are the objects that are $a$ s or $b \mathrm{~s}$, assuming no object is both $a$ and $b$.

Definition (16) introduces "identity" between $s / n$ operators, which itself is of category $s /(s / n),(s / n)$.

One technical remark: nominal definitions are supposed to have the conjunct " $A \varepsilon A$ " in the definiens, but the Definitions (13)-(15) do not contain it. Officially, it should be there, but for the sake of simplicity it can be dropped when the right-hand side of the definiens already entails it. The reader is free to re-write these definitions in the official format.

Now, we can move towards speaking about cardinalities. First, we have to make sure that, among those expressions of category $s / n, n$, we can distinguish those which are relations between individuals:

$$
\forall R[\operatorname{Rel}(R) \leftrightarrow \forall A, B(R(A, B) \rightarrow A \varepsilon A \wedge B \varepsilon B)]
$$

Next, we can define 1-1 relations and equicardinality:

$$
\begin{aligned}
& \forall R[\text { OneOne }(R) \leftrightarrow \operatorname{Rel}(R) \wedge \forall A, B, C, D((R(A, B) \wedge R(C, B) \rightarrow A=C) \wedge \\
& \wedge(R(A, B)\wedge R(A, D) \rightarrow B=D))], \\
& \forall a, b[\text { a eqc } b \leftrightarrow \exists R[\text { OneOne }(R) \wedge \\
& \forall A[\text { A } a \rightarrow \exists B(B \varepsilon b \wedge R(A, B))] \\
&\forall D[D \varepsilon b \rightarrow \exists C(C \varepsilon a \wedge R(C, D))]]] .
\end{aligned}
$$

Now, among expressions of category $s / n$, we can distinguish cardinality predicates: those that non-vacuously apply only to equicardinal names:

$$
\forall f[\operatorname{Card}(f) \leftrightarrow \exists a(f(a) \wedge \forall b(f(b) \rightarrow b e q c a))] .
$$

Furthermore, we define the successor functor of category $(s / n) /(s / n)$, which applied to an $s / n$ predicate $f$, yields another $s / n$ predicate, $\operatorname{succ}(f)$ :

$$
\forall f, a[\operatorname{succ}(f)(a) \leftrightarrow \exists A, b[E !(A) \wedge a \equiv A \oplus b \wedge f(b)]]
$$

(21) requires $\operatorname{succ}(f)$ to apply to $a$ just in case there is a $b$ to which $f$ applies and the extension of as consists of $b$ enriched with one other object. Strictly speaking, if $f$ is not a cardinality predicate, $\operatorname{succ}(f)$ will not be a cardinality predicate either. However, it is provable that successors of cardinality predicates are cardinality predicates:

$$
\operatorname{Card}(f) \leftrightarrow \operatorname{Card}(\operatorname{succ}(f))
$$

Simons [6] mentions this without a proof, but the reason why this holds is this: say for some $a, b$, $\operatorname{succ}(f)(a), \operatorname{succ}(f)(b)$. Call the result of throwing one object out of $a$ s $a^{\prime}$ (and similarly for $b$ ). Then, we should have $f\left(a^{\prime}\right)$ and $f\left(b^{\prime}\right)$, and so by the assumption that $f$ is a cardinality predicate, we have $a^{\prime} e q c b^{\prime}$. However, by construction, it also follows that $a$ eqc $b$. Argument in the other direction is analogous. 
Numerical predicates are easily definable:

$$
\begin{aligned}
& \forall a[0(a) \leftrightarrow N(a)], \\
& \forall a[1(a) \leftrightarrow E !(a)], \\
& \forall a[2(a) \leftrightarrow \operatorname{succ}(1)(a)], \\
& \forall a[3(a) \leftrightarrow \operatorname{succ}(2)(a)],
\end{aligned}
$$

and some easy claims about these follow:

$$
\begin{array}{r}
0(\Lambda), \\
\forall A[A \varepsilon A \leftrightarrow 1(A)], \\
\operatorname{Card}(0), \\
\operatorname{Card}(1), \\
1(a) \leftrightarrow \operatorname{succ}(0)(a) .
\end{array}
$$

It is also possible to define addition for predicates:

$$
\forall f, g, a[(f+g)(a) \leftrightarrow \exists b, c(a \equiv b \oplus c \wedge f(b) \wedge g(c))] .
$$

It then follows that adding cardinal predicates gives a cardinal predicate:

$$
\forall f, g[\operatorname{Card}(f) \wedge \operatorname{Card}(g) \rightarrow \operatorname{Card}(f+g)] .
$$

The converse fails for infinite cardinalities but holds restricted to finite ones. These notions can also be expressed in the language:

$$
\begin{aligned}
& \forall a[\inf (a) \leftrightarrow \exists b[b \subset a \wedge \neg a \subset b \wedge b e q c a]], \\
& \forall a[\operatorname{fin}(a) \leftrightarrow \neg \inf (a)],
\end{aligned}
$$

and the expected results about these notions are derivable in the system:

$$
\begin{aligned}
& \forall f[\operatorname{fin}(f) \rightarrow f \operatorname{in}(\operatorname{succ}(f))], \\
& \forall f[\inf (f) \rightarrow \inf (\operatorname{succ}(f))] .
\end{aligned}
$$

Now, how are natural numbers to be defined? Simons takes the standardly defined notion of the ancestral $\star R$ of a relation $R$. Then, start with the definition of successors among cardinal "numbers" and proceed to the definition of natural numbers. Keep in mind, however, that numerical variables in the system are of category $s / n$ :

$$
\begin{aligned}
\forall m, n[m \operatorname{folg} n \leftrightarrow \operatorname{Card}(m) & \wedge \operatorname{Card}(n) \wedge m \approx \operatorname{succ}(n)], \\
\forall n[\operatorname{Nat}(n) \leftrightarrow n & \approx 0 \vee n \star \operatorname{folg} 0] .
\end{aligned}
$$

How strong is the resulting arithmetic? One way to mimic the first-order language of standard arithmetic is to define higher-order predication copula. (One has to be careful here, as using a higher-order epsilon while trying to emulate set theory in Ontology is known to lead to trouble ([1] chapter 7).)

$$
f \hat{\varepsilon} G \leftrightarrow \exists a(f(a) \wedge G(f)) .
$$


Representing basic axioms of Peano Arithmetic (ignoring addition and multiplication) in the language yields:

$$
\begin{aligned}
& 0 \varepsilon \hat{E N a t,} \\
& \forall n[n \varepsilon ́ N a t \rightarrow \operatorname{succ}(n) \hat{\varepsilon} N a t], \\
& \forall n[n \varepsilon ́ N a t \rightarrow \neg \operatorname{succ}(n) \approx 0], \\
& \forall n, m[n \varepsilon ́ N a t \wedge m \varepsilon ́ N a t \wedge \operatorname{succ}(n) \approx \operatorname{succ}(m) \rightarrow n \approx m], \\
& \forall G, m[0 \dot{\varepsilon} G \wedge \forall n[n \varepsilon \dot{\varepsilon} N a t \wedge n \dot{\varepsilon} G \rightarrow \operatorname{succ}(n) \hat{\varepsilon} G] \wedge m \varepsilon \dot{\varepsilon} N a t \rightarrow m \varepsilon \dot{\varepsilon} G] \text {. }
\end{aligned}
$$

The system as it stands proves only (HP1) and (HP3). The rest of the axioms becomes derivable upon the addition of the axiom of infinity:

$$
\operatorname{Inf}(V) \text {. }
$$

\section{Neologicism}

\subsection{Background}

Abstraction Principles (APs), first used by Frege [7-9], have recently been brought back into foundational studies within neologicism [10-12]. APs are expressions of the form:

$$
f(\sigma)=f(\tau) \equiv \sigma R \tau,
$$

where $R$ is an equivalence relation, and $f$ is a newly introduced operator ( $\sigma$ and $\tau$ can be first- or higher-order variables).

Roughly speaking, on the neologicist approach, an AP is meant to fix the reference of abstract terms and explicate operation $f$, which, on the intended interpretation, assigns abstract objects to things that $\sigma$ and $\tau$ range over. For instance, Hume's Principle (HP) says that the number of one concept is the same as the number of another concept if and only if those concepts are equinumerous (a notion defined independently of the notion of number).

In his Treatise 1.3.1, Hume says:

We might proceed, after the same manner, in fixing the proportions of quantity or number, and might at one view observe a superiority or inferiority betwixt any numbers, or figures, especially where the difference is very great and remarkable. As to equality or any exact proportion, we can only guess at it from a single consideration, except in very short numbers, or very limited portions of extension, which are comprehended in an instant, and where we perceive an impossibility of falling into any considerable error. In all other cases we must settle the proportions with some liberty, or proceed in a more artificial manner. [...] There remain, therefore, algebra and arithmetic as the only sciences, in which we can carry on a chain of reasoning to any degree of intricacy, and yet preserve a perfect exactness and certainty. We are possest of a precise standard, by which we can judge of the equality and proportion of numbers; and according as they correspond or not to that standard, we determine their relations, without any possibility of error. When two numbers are so combined, as that the one has always an unite answering to every unite of the other, we pronounce them equal; and it is for want of such a standard of equality in extension, that geometry can scarce be esteemed a perfect and infallible science.

By introducing this principle, we are supposed to: fix references of expressions such as the number of $F^{\prime}$, determine an operation that assigns numbers to objects, and explicate our sortal concept of a number. Adding comprehension principle for concepts and Hume's Principle to second-order logic yields a consistent system, which allows for derivation of second-order Peano Arithmetic [13]. 
Neologicism is not devoid of challenges [14]. The neologicist would like to claim that APs they use are conceptually true. However, how can this claim be justified? One can not say that all APs are true because (within a sensible logical framework) certain APs (such as Basic Law V, which says that the extension of $F s$ is identical with the extension of $G$ s just in case $F$ and $G$ are predicates that apply to the same objects) lead to straightforward contradictions (within impredicative contexts). One can not require that all consistent APs are true because there is no consistency test for APs. What is worse, certain APs are separately consistent but mutually exclusive, and hence not all of them can be true. These and related problems give rise to the fairly open problem of finding sensible acceptability conditions of APs. All suggestions put forward so far are quite complicated, often requiring assumptions stronger than those provable in standard set theory, and there is no general agreement as to their plausibility and effectiveness. On top of that, as Incurvati [15] points out, even if there was a complicated criterion that neologicists would agree on, a person about to decide whether to accept given APs as the foundations of given mathematical theories would be in no position to know that those APs satisfy this criterion. After all, proving that they do requires resources stronger than the theories for which the subject is trying to find foundations.

More philosophical issues also arise. It is not clear that APs are capable of fixing references of abstract terms. Given a domain of non-abstract objects, no AP determines unambiguously the set of abstract objects that have to be added to this domain in order for the principle to hold. Even if we restrict ourselves to a certain cardinality of such an extended model, certain principles will still have non-isomorphic models, and all APs will be insensitive to permutations of abstract objects.

Say I introduce a new sort of objects, which I call "utopiec". I insist that these sorts of objects are correctly introduced by the following abstraction principle (the variables range over people):

$x^{\prime}$ s utopiec is the same as $y^{\prime}$ s utopiec iff $x$ and $y$ weigh the same.

Notice that the relation on the right-hand side is an equivalence relation. If the neologicist story is correct, the left side of any substitution of this principle entails the existence of a utopiec (just as the left-hand side of Hume's Principle can be used to prove the existence of numbers), whereas the right side does no such a thing. One way to go about showing the commitment of the left side is to observe that for any physical object $x, x$ weighs the same as $x$. One substitution of the principle under discussion is:

$x^{\prime}$ s utopiec is the same as $x^{\prime}$ s utopiec iff $x$ and $x$ weigh the same.

Since we have an equivalence and its right-hand side, we can deduce its left-hand side, formally $\operatorname{utopiec}(x)=\operatorname{utopiec}(x)$, from which, by existential instantiation, it follows that something is $x^{\prime} \mathrm{s}$ utopiec: $\exists y y=u \operatorname{topiec}(x)$, which, prima facie, carries ontological commitment to a utopiec.

If the ontological commitments of two sides of an equivalence differ, how can it be conceptually true? Even if we accept the principle, how does it help in determining whether a given object is a utopiec? Even if we were able to identify the sort of objects in question, how would we be able to assign them to appropriate objects?

A similar worry [16] is that it is unclear to what extent APs can be analytically or conceptually true, if what they do is allow for derivation of new existential statements. For instance, HP allows for inferring the existence of infinitely many numbers.

\subsection{APs within Ontology}

Quite interestingly, as Joray [17] observes, one can explicity define the cardinality of... operator within Ontology. It is of category $(s / n) / n$ : that is, applied to a name variable, it gives an operator that, applied to a name variable, yields a formula:

$$
\forall a, b[N o(a)(b) \leftrightarrow a e q c b] .
$$


The definition reads: the number of $a$ is ittributable to $b$ just in case $a$ and $b$ are equinumerous. In the system, it is now easy to derive a predicative version of Hume's Principle:

$$
\forall a, b[N o(a) \approx N o(b) \equiv a e q c b] .
$$

Similarly, we can define the extension of... operator by means of a definition which says that $b$ has the extension of $a$ just in case $a$ and $b$ s are the same objects:

$$
\forall a, b[\operatorname{Ext}(a)(b) \leftrightarrow a \equiv b] .
$$

By means of this definition, one can trivially prove a predicative variant of Basic Law V:

$$
\forall a, b[\operatorname{Ext}(a) \approx \operatorname{Ext}(b) \leftrightarrow a \equiv b] .
$$

In general, the following seems to hold:

Conjecture 1. For any relation definable in Ontology, if it is provable in Ontology that it is an equivalence relation, it is possible to introduce an appropriate operator, define it properly, and then prove the AP appropriate for it.

Proving the conjecture in the whole generality (i.e., about equivalence relations for all syntactic categories) will be tiring. Instead, let us restrict ourselves to equivalence relations applying to name variables.

Proposition 2. Consider the language of Ontology with the restriction that only variables of categories $s, s / n$ and $\frac{s / n}{n}$ can be used. Say we define a relation constant $R$ of category $s / n, n$ and $R$ is provably an equivalence relation. Then, it is possible to define a constant $f_{R}$ of category $\frac{s / n}{n}$ so that the AP corresponding to $R$ :

$$
\forall a, b\left[f_{R}(a) \approx f_{R}(b) \leftrightarrow a R b\right]
$$

becomes provable (mind (16)).

Proof. Indeed, define:

$$
\forall R, a, b\left[f_{R}(a)(b) \leftrightarrow a R b\right] .
$$

Now, the goal is to use (DefR) to derive (APR) using (16).

From right to left. Reason within the system. Suppose $a R b$. Take any $c$ for which $f_{R}(a)(c)$. By (DefR) $a R c$. By $a R b, a R c$, and the fact that $R$ is provably an equivalence relation, we have $f_{R}(b)(c)$. The argument from $f_{R}(b)(c)$ to $f_{r}(a)(c)$ is analogous. The choice of $c$ was arbitrary, and so we have:

$$
\forall c\left[f_{R}(a)(c) \leftrightarrow f_{R}(b)(c)\right],
$$

which by (16) means:

$$
f_{R}(a) \approx f_{R}(b)
$$

From left to right. Again, reason within the system. Say $f_{R}(a) \approx f_{R}(b)$. Since $R$ is an equivalence relation, $a R a$ and so $f_{R}(a)(a)$. By the assumption, we have $f_{R}(b)(a)$, and so $b R a$ by the fact that $R$ is an equivalence relation $a R b$.

\section{Discussion}

It does seem that the system is superior to that of PM. No axiom of reducibility is needed, and so the natural language phenomenon of numerical terms sometimes being used as predicates of 
predicates and sometimes being subjects of other predicates is nicely preserved. There are, however, further issues that deserve discussion.

\subsection{Unity}

One problem of type-theoretic foundations of arithmetic that remains is this. With proper caution, numerical terms can be introduced at each level of the hierarchy of predication (on higher levels they have to count not individuals, but something else-whatever is used in the interpretation of higher-order variables - up to coextensivity). Then, however, it seems that there are infinitely many copies of each natural number at different levels, and there is no way of identifying them within the system.

The Leśniewskian scholars are quite optimistic:

As it has been shown by Nadine Gessler, type (or categorial) ambiguity is not needed to warrant the unity of all the higher-degree arithmetics, which can be developed in Ontology. ([17], p. 162)

Indeed, Gessler ([18], pp. 72-73) suggests that, because at each higher-order level it is possible to define the corresponding higher-order epsilon, in the style of (32), the system is developed "without systematic ambiguity".

This assessment seems too optimistic for at least two reasons. For one thing, just because one can define higher-order epsilons does not remove the problem of there being different numerical terms at different higher-order levels, to which different higher-order epsilons are to be applied. For another, Gessler suggests that whatever unity is gained, it is provided by means internal to the system because higher-order epsilons are defined within it. However, this is too hasty: explaining why all those definitions count as definitions of one and the same epsilon at different levels, and describing how for all levels such definitions are to be given is a task that cannot be performed within the system.

Simons ([6], p. 238) suggests that the path to re-gaining the univocity of numerical terms lies in treating multitudes as objects, but he only gestures towards this approach without developing it, and it is known that doing so, even within Leśniewskian framework, is quite a challenge [19].

\subsection{Quantification and Ontological Commitment}

Simons ([6], p. 235) (as people sympathetic to Leśniewski's systems usually do) insists that the system has "no ontological implications" - after all, the system does not prove that at least one object exists $(\exists A, a A \varepsilon a)$.

Similarly, Joray ([17], p. 161) comments:

... the fact that abstraction's results are not designated as objects preserves the ontological neutrality of logic. Theorems of Leśniewski's calculus are logically true in the sense they are true in all domains, including the empty one.

In the same vein, Gessler ([18], p. 67) explains:

The source of this neutrality lies in the methods of interpretation of the quantification. Neither referential, nor substitutional, the quantification is of categorial nature. It applies to variables of any semantic category, be it propositional, nominal or functorial, while its interpretation eludes the question of ontological commitment regarding the existence of objects constituting the possible significances of the related variables. Where there is the question of semantic categories, there is by no means a question of ontological categories. To each category is associated a quantification domain which must be understood as the possibilities of extensional significances falling under the category in questions. The form [RU: my notation] $\forall v A(v)[\ldots]$ must be read thus: "whatever the extensional significance alloted to the variable $v$-respectively for any extensional significance allotted to the variable $v$-it is the case that $A(v) . "$ 
Now, the problem is that claiming that numerical terms within the system are not objectual does not explain how they are to be interpreted. Similarly, saying that quantification is neither referential nor substitutional does not explain how it is to be understood. Just because the domain of individuals does not have to be non-empty for the theorems of Ontology to hold, it does not follow that it is clear that Ontology has no ontological commitment to objects other than individuals (that is, that assuming the truth of the theorems of Ontology, one is not committed to the existence of anything). What do higher-order variables range over? How should we interpret quantifiers? Gessler's suggestion that the quantification domain for a given syntactic category consists of the possibilities of extensional significances needs to be elaborated to make it clear that a nominalist can use such domains in their semantics of a formal language.

Similarly, Joray ([20], p. 8), when it comes to explaining higher-order quantifiers, says that quantifiers range over sets of functions. Mathematically, this is all fine, but if one at the same time wants to say that Ontology has no ontological commitment, more has to be said about how the nominalist is supposed to think about higher-order quantifiers.

I think Gessler's suggestion is correct-some modal aspect has to be brought into play for the nominalist to make sense of higher order quantification. The notion of ontological commitment has been discussed and the modal intuition intuition has been further developed into full-blown modal semantics for higher-order quantification in ([1], Chapter 8), [21] and [22]. Since here I am unable to say anything superseding what is already in those papers, let us move on (see however also $[23,24]$ for a deeper discussion of the notion of ontological commitment in this context).

\subsection{Troubles with Infinity}

For the metaphysically parsimonious mind, another problem arises when it turns out that most of the (representations of the) standard axioms of arithmetic are unprovable in the system, unless we conjure the assumption that the domain is infinite. You can try to massage this assumption into something that a nominalist might live with on a sunny day.

The axiom of infinity is not an empirical statement concerning the world, but a hypothesis specifying the kind of idealization through which we apply arithmetic to specific concrete situations. ([17], p. 163)

After all, the infinity of concrete objects is not per se offensive to the nominalist. If, however, you're motivated to be a nominalist by epistemological considerations, then just as you might think, there are no good reasons to accept the existence of abstract objects, and you might be worried about there being no good reasons to accept the existence of infinitely many objects, whatever their nature. This is the issue I will try to handle further in this paper.

(By the way, note that the problem of infinity does not only rise in the philosophy of mathematics, but also in the philosophy of physics. At least prima facie contemporary physics requires a non-denumerable actual infinity of objects, given the use of real number theory in physics.)

Another kind of issue arises when one tries to avoid the problem with postulating that the domain of objects is infinite by applying the Fregean bootstrapping strategy. In this approach, one first proves the existence of number zero by taking an empty concept and showing that its number exists, then the existence of number one follows by taking a concept which applies exactly to number zero and showing that its number exists, and so on. This trick does lead to the existence of an infinite class of witnesses to make the axioms of standard arithmetic true. However, I find this approach deeply deficient when it comes to explaining the applicability of arithmetic to real-world issues.

So, what is left to do? In what follows, we will try to avoid both kinds of worries by (i) avoiding the artificiality of bootstrapping by keeping considerations at one level of semantic hierarchy, following the intuition that numbers were introduced to count objects, whatever they are, and not to count lower numbers; and (ii) avoiding the ontological commitment to infinity by requiring only that, independently of how many objects there are, there could be more. This means that the 
price I will pay for avoiding the challenges mentioned above will be the introduction of modalities into the theory. (Actually, whether it even is a price, is not obvious. Shapiro insists it is [25]. I disagree [21].) To be fair, however, I have no idea how else one could make sense of the distinction between potential and actual infinity, which will be of crucial importance in what follows, and which has been present in the philosophy of mathematics since Aristotle.

\section{Actual and Potential Infinity}

\subsection{Background}

Let us ignore some of the issues discussed: let us not worry about the unity of the framework and focus on the numerical terms on the first level of hierarchy where they become available. Let us also not care too much now about the nominalistic acceptability of higher-order quantification. The issue has been extensively discussed elsewhere-let us simply assume it is available to the nominalist (with the proviso that a more detailed account not only is due, but has been given [21,22].) Thus for now, we will be interested only in the predicative arithmetic obtainable over a domain of objects by means of tools provided by Ontology and fairly natural modification thereof.

What is the alternative to simply postulating that infinitely many objects actually exist, if one wants to make the standard arithmetic true? Well, at the philosophical level, one can try to conjure the idea of the distinction between actual and potential infinity. While the distinction is brought up every now and then in philosophical discussions, there are not too many attempts to use this notion in a formal setting in the foundations of classical mathematics-partially because explicating the notion of potential infinity formally is a bit of a challenge, and partially because the distinction is considered responsible for the development of intuitionistic mathematics, and so the idea that using the distinction in the foundations of classical mathematics seems foreign. This is also partially because proceeding in this direction requires some use of modalities, and using modal logic in the foundations of mathematics is far from mainstream.

There is a research program that, at least partially, goes in this direction. It is the study of arithmetic over finite but potentially infinite models, pursued by Marcin Mostowski and some of his students in Warsaw [26,27], and further applied in philosophical research in the foundations of computability [28]. The basic notions on this approach are that of an FM-domain ("FM" coming from finite model) and that of SL-semantics ("SL" coming from sufficiently large).

Roughly, an FM-domain is a family of finite initial segments of arithmetical models, and a formula is $S L$-satisfied in an FM domain if there is a size $n$ of a domain such that that a formula is satisfied in all models of size $n$ and larger belonging to the FM-domain. To assess a formula in an FM-domain, one has to assess it point-wise, in each particular finite model, and to check if the formula is satisfied in all models large enough to satisfy it. In each particular point, relation symbols from the language of arithmetic are interpreted as restrictions of the usual relations to the finite domain at hand, and function symbols are interpreted normally as long as the value belongs to a given initial segment in the standard model, taking the maximal element otherwise.

In a sense, modalities are swept under the carpet, but they are there. No modal operator is present in the object language, but the whole FM-domain can be seen as a collection of all possible finite worlds. Then, assessment at each point is the assessment of a formula in a finite world, while assessment in the whole FM-domain is the evaluation of a formula in a potentially infinite domain.

Given the way the semantics are constructed, there are some artificially-sounding side-effects. The main reason for this is that semantics, instead of remaining neutral on whether actual infinity exists, already build in the assumption that any particular possible world is finite. As a result, those formulas which are true in all finite worlds come out true, and the resulting set of FM-valid formulas is not the set of standard arithmetical truths. For instance, in every FM-domain, it is true that the greatest number exists because each particular finite initial segment belonging to it indeed does contain the greatest number. This is one issue that we would like to overcome. 
Another issue is that, while the approach is finitist, it is by no means logicist. Numbers (albeit finite) are simply taken to be members of points in an FM-model and that is it. We, on the other hand, would like to preserve the logicist flavor of Leśniewskian arithmetic, while still approaching the issue in terms of potential infinity. Let us get to work.

\subsection{Modalizing Quantifiers}

Say we want to approach the language of Ontology (and a modal extension thereof) using the standard set-theoretic tools of model theory.

Definition 3. A model $\mathcal{D}$ for the standard language of Ontology is composed of a (possibly empty) domain of objects $D$ and quantification domains for variables of different categories. The range of quantification of type $n$ (that is, the range of name variables)—-marked as $D_{n}$-is $\mathcal{P}(D)$, the range of quantification of type $s-D_{s}-$ is $\{0,1\}$, and generally the range $D_{\tau /\left(\chi_{1}, \ldots, \chi_{n}\right)}$ of quantification of type $\tau /\left(\chi_{1}, \ldots, \chi_{n}\right)$ is the set of all functions from $D_{\chi_{1}} \times \cdots D_{\chi_{n}}$ to $D_{\tau}$. Defined constants of type $\tau$ pick appropriate elements of $D_{\tau}$ to make the definitions hold. Such models will be called points in what follows. A point-valuation $v$ assigns to any variable of a given category $\tau$ some element of $D_{\tau}$. $\mathcal{D}, v \models a \varepsilon b$ just in case $v(a)$ is a singleton and $v(a) \subseteq v(b)$, and the clauses for the rest of the standard language are obvious.

Now, however, we would like to somehow be able to talk about potential infinity, and so a modal aspect needs to be introduced. This will be done both on the level of models, and on the level of the object language. Let us start with the former.

Definition 4. A set of points $\mathcal{W}$ (see Definition 3) is an $\infty$-frame just in case, for any point $\mathcal{D} \in \mathcal{W}$, there is a $\mathcal{D}^{\prime} \in \mathcal{W}$ such that the following condition is satisfied:

$$
D \subseteq D^{\prime} \wedge \overline{\overline{D^{\prime}}}>\overline{\bar{D}}
$$

The condition says that the domain of $\mathcal{D}$ is contained in the domain of $\mathcal{D}^{\prime}$ and that the cardinality of $D^{\prime}$ is greater than that of $D$. If $D$ and $D^{\prime}$ are in this relation, we will say that $D^{\prime}$ extends $D$. Requirement (Ext) has been introduced to capture the idea that, however many objects there are, there always could be more.

If all points in an $\infty$-frame are finite, we call it an F-frame. We say that $\mathcal{D}^{\prime}$ is accessible from $\mathcal{D}$ $\left(\mathcal{D} R \mathcal{D}^{\prime}\right)$ just in case $D \subseteq D^{\prime}$. In the case of F-frames, requirement (Ext) boils down to $D \subset D^{\prime}$.

Why would we focus on F-frames, though? Didn't the restriction to finite initial segments lead to trouble with obtaining all and only arithmetical truths in FM-domains? Will it not lead to trouble now? Well, on one hand, the requirement is a bit too strong: after all, mathematics should not decide or assume an answer to the question whether the number of things in the world is finite or not. From this perspective, no cardinality requirement should be put on the size of points. On the other hand, the essential question now is whether standard arithmetic can be regained even if the world is (and has to be) finite. From this perspective, it also seems interesting to see whether the focus on F-frames will ruin things or not.

Now, let us extend the language of Ontology with modal quantifiers $\Sigma$ and $\Pi$ (binding variables of arbitrary categories). Intuitively, ' $\Sigma a \phi(a)^{\prime}$ ' reads 'There could be enough objects so that for some $a$, $\phi(a)^{\prime}$ and ' $\Pi a \phi(a)^{\prime}$ 'reads 'however many more objects there could be, it still would be the case that for any $a, \phi(a)^{\prime}$. Call the set of variables of the language be Var.

Definition 5 (valuation, satisfaction). A valuation in a frame $\mathcal{W}$ is a total function from $W \times \operatorname{Var}$ such that if $\tau$ is a variable of category $t$, for any $\mathcal{D} \in \mathcal{W}$, we have $v(\mathcal{D}, \tau) \in D_{t}$, so that valuation interprets variables as elements of domains of appropriate type. Satisfaction clauses for Boolean connectives 
and classical quantifiers are standard. $\mathcal{D}, v \mid=\Sigma \tau \phi$ just in case there is a $\mathcal{D}^{\prime} \in W$ and a valuation $v^{\prime}$ such that: (i) $\mathcal{D} R \mathcal{D}^{\prime}$, (ii) for any variable $\chi$ other than $\tau, v^{\prime}\left(\mathcal{D}^{\prime}, \chi\right)=v(\mathcal{D}, \chi)$ (recall that the values of $v$ from $\mathcal{D}$ are still available in $\mathcal{D}^{\prime}$ ), and (iii) $\mathcal{D}^{\prime}, v^{\prime} \models \phi$. $\Pi \tau \phi$ is defined by $\neg \Sigma \tau \neg \phi$. Truth is defined as satisfaction under any valuation.

Now, the question is, whether once we replace standard quantifiers with those modalized quantifiers, arithmetic can be regained. We are after these formulas:

$$
\begin{aligned}
& \text { 0éNat， } \\
& \Pi n[n \varepsilon ́ N a t \rightarrow \operatorname{succ}(n) \varepsilon=\hat{N a t}], \\
& \Pi n[n \varepsilon ́ N a t \rightarrow \neg \operatorname{succ}(n) \approx 0], \\
& \Pi n, m[n \varepsilon \hat{E} N a t \wedge m \varepsilon \dot{N a t} \wedge \operatorname{succ}(n) \approx \operatorname{succ}(m) \rightarrow n \approx m] \text {, } \\
& \Pi G, m[0 \tilde{\varepsilon} G \wedge \forall n[n \varepsilon \hat{\varepsilon} \text { at } \wedge n \varepsilon \dot{\varepsilon} G \rightarrow \operatorname{succ}(n) \hat{\varepsilon} G] \wedge m \varepsilon \dot{\varepsilon} \text { at } \rightarrow m \varepsilon \dot{\varepsilon} G],
\end{aligned}
$$

where all quantifiers in the definitions of the defined terms occurring in these formulas have been replaced by their modal counterparts.

Theorem 6. For any $F$-frame $\mathcal{W}$, for any $\mathcal{D} \in \mathcal{W}$, (MHP1)-(MHP5) are true in $\mathcal{D}$.

Proof. For (MHP1), notice that $0 \varepsilon \hat{N}$ at reads $\Sigma a(0(a) \wedge N a t(a))$. Because accessibility is reflective, it is enough to show that in any point in the frame, $\exists a(0(a) \wedge N a t(0))$. This reads:

$$
\exists a(0(a) \wedge 0 \approx 0 \vee 0 \star f o l g(0)),
$$

that is:

$$
\exists a(0(a) \wedge(\Pi(z)(0(z) \equiv 0(z)) \vee 0 \star f o l g 0))) .
$$

The left disjunct in the bracket is obviously true, which makes the right conjunct true. For the witness of the existential quantifier, simply take $\varnothing$, which belongs to any $D_{n}$.

For (MHP2), notice that it reads:

$$
\Pi n[\Sigma a((a) \wedge \operatorname{Nat}(n)) \rightarrow \Sigma b(\operatorname{succ}(n)(b) \wedge \operatorname{Nat}(\operatorname{succ}(n))] .
$$

Suppose $\mathcal{D} R \mathcal{D}^{\prime}$ and $\mathcal{D}^{\prime}, v \mid=\Sigma a(n(a) \wedge N a t(n))$, where $v$ differs in $\mathcal{D}^{\prime}$ from $\mathcal{D}$ at most on $n$. This means that $n$ applies to all equinumerous elements of $D_{n}^{\prime}$ of a certain finite size, and that $D_{n}^{\prime}$ indeed contains at least one element of that size.

Then, by (Ext), there is a $\mathcal{D}^{\prime \prime}$ accessible from $D^{\prime}$ whose $D^{\prime \prime}$ contains a new element $x$ not occurring in $D^{\prime}$. Take a valuation $v^{\prime}$ which differs from $v$ in $\mathcal{D}^{\prime \prime}$ from $\mathcal{D}^{\prime}$ at most on $b$, such that $v^{\prime}\left(\mathcal{D}^{\prime \prime}, b\right)=v(a) \cup\{x\}$. Then, clearly $\operatorname{succ}(n)(b)$ and $\operatorname{Nat}(\operatorname{succ}(n))$. This means $\mathcal{D}^{\prime}, v \mid=\sum b(\operatorname{succ}(n)(b) \wedge \operatorname{Nat}(\operatorname{succ}(n))$, and so $\mathcal{D}, v \models(37)$.

(MHP3) says that in any world $\mathcal{D}^{\prime}$ accessible from $\mathcal{D}$, where $n$ applies only to equicardinal elements of $D_{n}^{\prime}$ of a certain finite size, in any $\mathcal{D}^{\prime \prime}$ accessible from $\mathcal{D}^{\prime}, \operatorname{succ}(n)$ will not apply to $\varnothing$. This is true because, by (Ext), $D^{\prime \prime}$ will contain a new element not present in $D^{\prime}$, which will be in the denotation of an element $b$ of $D_{n}^{\prime}$ to which $\operatorname{succ}(n)$ will apply. Since successors of cardinal numbers are cardinal numbers, $\operatorname{succ}(n)$ will apply only to elements of $D_{n}^{\prime}$ equinumerous with $b$, none of which will be $\varnothing$.

(MHP4) requires, roughly, that in any accessible world $\mathcal{D}^{\prime}$ in which in $D_{n}^{\prime}$ there are $n$ and $m$ elements, if in any world $D^{\prime \prime}$ accessible from $D^{\prime} \operatorname{succ}(n)$ and $\operatorname{succ}(m)$ apply to the same elements of $D_{n}^{\prime \prime}, m$ and $n$ apply to the same elements of $D_{n}^{\prime}$. The only reason why this could fail is if in all possible worlds accessible from $\mathcal{D}^{\prime}$, but (Ext) excludes this possibility. 
Finally-(MHP5). Consider the content of $\mathcal{D}, v \models 0 \varepsilon \hat{\varepsilon}$. This says there is a $\mathcal{D}^{\prime}$ accessible from $\mathcal{D}$ such that $\mathcal{D}^{\prime}, v^{\prime}=0(a) \wedge G(0)$, where $v^{\prime}$ differs from $v$ at most on $a$. This means that already $\mathcal{D}, v \models G(0)$. With this in mind, the simplified content of (MHP5) reads: if 0 is $G$, and for any natural $n$, if $G(n)$, then $G(\operatorname{succ}(n))$ would be true, and then for all natural $m, G(m)$. This is true because of the way Nat was defined.

Notice also that in any F-frame, the claim that was problematic in FM-domains ('the greatest number exists') turns out false. $w \models \Sigma n \Pi m n \geq m$ would mean that there is a $w^{\prime}$ accessible from $w$, where for some $n$, for any $w^{\prime \prime}$ accessible from $w^{\prime}$ for any $m \in w^{\prime \prime}, n$ is greater than $m$. However, this is false because there is a world $u$ accessible from $w^{\prime}$ that has more objects than $w^{\prime}$, and so, in $u$, there is a number greater than any number that existed in $w^{\prime}, n$ included.

\section{Leśniewski's Arithmetic}

Now, for the sake of completeness, let us take a look at the historical question of what Leśniewski's original arithmetic looks like.

\subsection{Remarks}

Most of what Leśniewski wrote did not have much to do with arithmetic. However, in Warsaw, he taught three courses related to the topic: Foundations of Arithmetic (1920-1923), Primitive Terms of Arithmetic (1928/1929) and Inductive Definitions (1933/1934). Student lecture notes from the second and the third survived. The material on inductive definitions is not too exciting (it is about finding explicit definitions for inductive definitions over arithmetic). The only piece of work to rely on is Chapter four of [29], titled Primitive Terms of Arithmetic.

As Simons ([6], p. 228) observes, this piece is...

... deeply disappointing, in the sense that it uses very little of the resources of Leśniewski's own most important logical system, Ontology. It consists simply in a formulation in Leśniewski's language of Peano's ideas about the natural numbers, taking these to be individuals of a certain kind, the natural numbers.

I will, however, describe both Leśniewski's arithmetic and the results that Leśniewski's has proven in and about it. For one thing, this is what historical completeness requires. For another, the original is not very user friendly - the paper is on pages 129-152, contains 376 words out of which 112 are the occurrences of the word Thesis ( \pm 15 , given how boring the counting was). The rest are just formulas in Leśniewski's own idiosyncratic notation. Thus, a more accessible presentation might be useful.

There is also a more important reason to develop a more accessible presentation: putting it in a main-stream format allows one to use modern tools to study the properties of the system.

\subsection{Axioms and the Second-Order Translation}

The language of Leśniewski's arithmetic is built over Ontology. Its specific terms are: $1, n a t, S q,+, \times,>$ with their obvious interpretations: number one, natural number, successor, addition, multiplication, and being greater than. In my presentation, I will use a simplified second-order language, whose primitive non-logical symbols will comprise $1, N, s,+, \times,>$ with their obvious interpretations.

While later the fact that Ontology is a free logic will matter, it does not matter as far as we investigate Leśniewski's arithmetic because it already contains the axiom requiring number one to exist: 
For the sake of streamlining of the system and making it accessible to the reader, (A1) can be written as:

$$
N(1)
$$

The second axiom requires that successors of natural numbers are natural numbers:

$$
\forall A[\text { Acnat } \rightarrow S q(A) \varepsilon n a t]
$$

which under our translation (translations of axioms will be marked with ') yields:

$$
\forall x[N(x) \rightarrow N(s(x))]
$$

Notice that Leśniewski's development does not use quantification other than second-order, and so in the translation using a second-order language is sufficient.

The third axiom says that one is not a successor of any number (I will put the translation right after the original):

$$
\begin{array}{r}
\forall A[\text { Asnat } \rightarrow \neg S q(A)=1], \\
\forall x[N(x) \rightarrow s(x) \neq 1] .
\end{array}
$$

The fourth axiom says that no two distinct numbers have the same successor:

$$
\begin{array}{r}
\forall A, B[\text { Acnat } \wedge \text { Benat } \wedge S q(A)=S q(B) \rightarrow A=B], \\
\forall x, y[N(x) \wedge N(y) \wedge s(x)=s(y) \rightarrow x=y] .
\end{array}
$$

Since the translation is obvious (and definitely too obvious to deserve a full proper definition), I will now just give the result, assuming the original axioms can be easily recovered, if need be. The next axiom is the axiom of induction, which is the only one in which Leśniewski uses higherorder quantification:

$$
\forall P \forall x[P(1) \wedge \forall y(N(y) \wedge P(y) \rightarrow P(s(y))) \wedge N(x) \rightarrow P(x)]
$$

The next two axioms introduce addition in the standard manner:

$$
\begin{array}{r}
\forall x[N(x) \rightarrow x+1=s(x)] \\
\forall x, y[N(x) \wedge N(y) \rightarrow x+(y+1)=(x+y)+1]
\end{array}
$$

Two further axioms introduce multiplication:

$$
\begin{array}{r}
\forall x[N(x) \rightarrow x \times 1=x], \\
\forall x, y[N(x) \wedge N(y) \rightarrow c \times(y+1)=(x \times y)+x] .
\end{array}
$$

The next two axioms pertain to the > relation:

$$
\begin{aligned}
\forall x, y[N(x) \wedge N(y) & \wedge x>y \equiv \exists z(N(z) \wedge x=y+z)] \\
& \forall x, y[N(x) \wedge N(y) \rightarrow x+y>x] .
\end{aligned}
$$

Finally, the last axiom-called A12 — of which Leśniewski remarks that it "is not a thesis of Peano Arithmetic" says that successors are always natural numbers:

$$
\forall x, y[y=s(x) \rightarrow N(y)]
$$




\subsection{Leśniewski's Theorems within Arithmetic}

Most of the lecture notes consists of formal and rather boring proofs of unsurprising arithmetical facts. Theorems $\left(\mathrm{T}^{\prime}\right)-\left(\mathrm{T} 6^{\prime}\right)$ are simple reformulations of the axioms and observations about the scope of $N$ :

$$
\begin{array}{r}
\forall x[\forall y(N(y) \wedge P(y) \rightarrow P(y+1)) \wedge N(x) \wedge P(x) \rightarrow P(x+1)], \\
\forall x[N(x) \rightarrow N(x+1)], \\
N(1+1), \\
N(x) \rightarrow \neg(x+1=1), \\
\forall x, y[N(x) \wedge N(y) \wedge x+1=y+1 \rightarrow x=y], \\
\forall x[P(x) \wedge \forall y(N(y) \wedge P(y) \rightarrow P(y+1)) \wedge N(x) \rightarrow P(x)] .
\end{array}
$$

Arithmetical proofs in the rest of Leśniewski's paper follow the general pattern of (i) defining an appropriate symbol expressing the property to be used in the inductive proof; and (ii) using the symbol in inductive argument to prove a claim. To give one example, $\left(\mathrm{D}^{\prime}\right)$ defines the property $\alpha \beta$ of being an $x$ such that adding $x$ to a natural number yields a natural number (notice that I dropped the first conjunct in the definiens, officially required by (n-Def) — this is because once we move to language of a logic that is not free, the existence assumption is in the background for free):

$$
\forall x[\alpha \beta(x) \equiv \forall y(N(y) \rightarrow N(y+x))]
$$

Then, Leśniewski runs induction to show that $\alpha \beta$ is coextensive with $N$ and in effect, $N$ is closed under addition (T10 of the original).

Similar moves are used to prove:

- that the addition of one is commutative (T13),

- and so is addition in general (T25, T27),

- that addition is associative (T17),

- that if the result of adding the same number to two numbers is the same, so are these two numbers (T21),

- that adding a natural number (recall, one is the least natural number in the system) to a number yields a different number (T35),

- that multiplying by one does not change the number (T46),

- that natural numbers are closed under multiplication (T50),

- that if the result of multiplying two numbers by one and the same number is the same, so are these two numbers (T57),

- that multiplication is commutative (T62),

- and that multiplication is associative (T67).

Then, a series of theorems about the ordering relations follows. Since these are historically slightly more interesting, let us list the key ones. Here, nothing interesting hangs on the restriction of quantifiers to natural numbers, so the formulae are to be read as if quantifiers were restricted to $N$ (also, mind that one is the least natural number in this setting): 


$$
\begin{array}{r}
\forall x \neg x>x, \\
\forall x, y, z(z=x+y \rightarrow z>x), \\
\forall x(x=1 \vee x>1), \\
\forall x, y(x=y \vee y>x \vee x>y), \\
\forall x, y, z(x>y \wedge y>z \rightarrow x>z), \\
\forall x, y, z(x>y \rightarrow x+z>y+z), \\
\forall x \neg 1>x, \\
\forall x, y, z(x>y \rightarrow x \times z>y \times z), \\
\forall x, y(x>1 \rightarrow y \times>x), \\
\forall x, y(y \times=x \vee y \times x>x) .
\end{array}
$$

\section{Remarks about the System}

The way Leśniewski formulated the system leaves certain questions unanswered. For one thing, Leśniewski remarks that $S q$ can be defined by:

$$
\begin{aligned}
\forall A, B[A \varepsilon S q(B) & \equiv \text { Asnat } \wedge \text { Benat } \wedge A \varepsilon B+1], & & \left(\operatorname{Def}_{S q}\right) \\
\forall x, y[x=s(y) & \equiv N(x) \wedge N(y) \wedge x=y+1] . & & \left(\operatorname{Def}_{S q}^{\prime}\right)
\end{aligned}
$$

Given Leśniewski's view on definitions [5], this should mean that, by means of this definition, all axioms and theorems involving $s$ should be derivable from $\left(\operatorname{Def}_{S q}^{\prime}\right)$ together with those axioms which do not involve $s$. This, however, does not seem to work very well given that $\left(\operatorname{Def}_{S q}^{\prime}\right)$ defines successor function in terms of adding one, while $\left(\mathrm{A}^{\prime}\right)$ defines adding one in terms of the successor function. Perhaps, all that Leśniewski meant was that, had we prior understanding of adding one, we could define the successor function by means of $\left(\operatorname{Def}_{S q}^{\prime}\right)$, but no understanding of +1 independent of $s$ is given in the paper.

Another interpretation is that we could simply replace all occurrences of $x=s(y)$ in the axioms with $x=(y+1)$, and then use the axioms together with $\left(\operatorname{Def}_{S q}^{\prime}\right)$ to derive all the usual theorems about s. However, then, $\left(\mathrm{A}^{\prime}\right)$ becomes $\forall x[N(x) \rightarrow x+1=x+1]$, which is not as informative as it should. However, let us move on.

Page 141 of [29] also contains the following remark:

The Axiom A12 $\star$ restricts the scope of the term 'Sq.' Without this restriction, the axiom system of Peano arithmetic is not sufficient.

The question is, how do we know that (A12') is not already derivable thanks to the induction axiom? After all, in the standard setting, second-order induction ensures categoricity and excludes non-standard successors. Well, here the difference is that, in the standard setting, the whole domain is the range of quantification occurring in the axioms, whereas, in the current system, pretty much all of the axioms are restricted to the extension of $N$, and no assumption is made about the domain not containing Julius Caesar or rabbits. In fact, we can provide an independence proof for the claim:

Theorem 7. (A12') is independent of the remaining axioms.

Proof. We just need to define a model satisfying $\left(\mathrm{A} 1^{\prime}\right)-\left(\mathrm{A} 11^{\prime}\right)$ but not $\left(\mathrm{A} 12^{\prime}\right)$. Take the domain to be positive real numbers starting with one, interpret 'one' as number 1 , take the successor symbol to be the successor function defined for all real numbers as the result of adding one, and interpret addition, multiplication and greater-than symbols as addition, multiplication and being greater than for the real numbers belonging to the domain. Interpret $N$ as the set of all natural numbers (starting with 


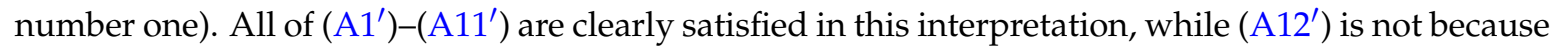
there are real numbers that are successors without being natural numbers.

Isn't it a terrible omission not to include (A12') among the standard axioms of Peano arithmetic then? Not really because once we restrict its content to natural numbers, it is easily derivable.

Theorem 8. The following restriction of (A12') is derivable from (A1')-(A11'):

$$
\forall x[N(x) \rightarrow(y=s(x) \rightarrow N(y))]
$$

Proof. Work within the system. Let $x$ be arbitrary and assume $N(x)$. By $\left(\mathrm{A} 2^{\prime}\right)$, we have $N(s(x))$, and so for $y=s(x)$, we have $N(y)$.

Theorem 8 shows that the underivability of $\left(\mathrm{A} 12^{\prime}\right)$ is a rather uninteresting result of the assumptions made about the domain, not a deep mathematical gap in the standard axiomatizations of Peano Arithmetic.

Another thing to observe is that $\left(\mathrm{A} 11^{\prime}\right)$ is redundant given $\left(\mathrm{A} 1^{\prime}\right)-\left(\mathrm{A} 10^{\prime}\right)$ :

Theorem 9. (A11') is derivable from $\left(\mathrm{A} 1^{\prime}\right)-\left(\mathrm{A} 10^{\prime}\right)$.

Proof. Work within the system.

$$
\begin{aligned}
& \text { 1. } N(x) \wedge N(y) \\
& \text { 2. } x+y=y+x \\
& \text { Assumption } \\
& \text { 3. } N(x) \wedge x+y=y+x \\
& \text { 4. } \exists z[N(z) \wedge x+y=y+z] \\
& \text { 5. } x+y>x \\
& \text { Induction and addition axioms } \\
& \text { Logic, 1, } 2 \\
& \text { Logic, } 3 \\
& \left(\mathrm{~A} 10^{\prime}\right), 4
\end{aligned}
$$

Acknowledgments: This research was supported by the Research Foundation Flanders. I am also grateful to Pawel Pawlowski, Michał Tomasz Godziszewski, Øystein Linnebo and two anonymous referees for their comments on earlier drafts of this paper.

Conflicts of Interest: The author declares no conflict of interest.

\section{References}

1. Urbaniak, R. Leśniewski's Systems of Logic and Foundations Of Mathematics; Springer-Verlag: Berlin, Germany, 2013.

2. Leśniewski, S. O Podstawach Matematyki, Rozdział X: Aksjomatyka Ogólnej Teorji Mnogości Pochodząca z r. 1921. Rozdział XI: O Zdaniach 'Jednostkowych' Typu 'Aeb'. Przeglad Filozoficzny 1931, 34, 142-170.

3. Leśniewski, S. Stanisław Leśniewski. Collected Works (Two Volumes); Surma, S., Srzednicki, J., Barnett, D.I., Eds; Kluwer Academic Publishers: Dordrecht, The Netherlands, 1991.

4. Urbaniak, R. The Non-Standard Interpretations of the Axiomatic Basis of Lesniewski's Ontology. Australas. J. Log. 2006, 4, 13-46.

5. Urbaniak, R.; Hämäri, K. Busting a Myth about Leśniewski and Definitions. Hist. Philos. Log. 2012, 33, 159-189.

6. Simons, P. Arithmetic in Leśniewski's Ontology. In The History and Philosophy of Polish Logic: Essays in Honour of Jan Woleński; Mulligan, K., Kijania-Placek, K., Placek, T., Eds.; Palgrave Macmillan UK: London, UK, 2014; pp. 227-241.

7. Frege, F. Die Grundlagen der Arithmetik: Eine Logisch-Mathematische Untersuchung über den Begriff der Zahl; W. Koebner: Breslau, Poland, 1884.

8. Frege, F. Grundgesetze der Arithmetik; Verlag Hermann Pohle: Jena, Germany, 1893; Volume (band 1).

9. Frege, F. Grundgesetze der Arithmetik; Verlag Hermann Pohle: Jena, Germany, 1903; Volume (band 2).

10. Hale, B.; Wright, C. The Reason's Proper Study. In Essays Towards a Neo-Fregean Philosophy of Mathematics; Oxford University Press: Oxford, UK, 2001; pp. 1-27. 
11. Wright, C. Frege's Conception of Numbers as Objects; Aberdeen University Press: Aberdeen, UK, 1983.

12. Zalta, E. Abstract Objects: An Introduction to Axiomatic Metaphysics; D. Reidel: Dordrecht, The Netherlands, 1983.

13. Boolos, G. The Consistency of Frege's Foundations of Arithmetic. In On Being and Saying: Essays for Richard Cartwright; MIT Press: Cambrige, MA, USA, 1987.

14. Fine, K. The Limits of Abstraction; Clarendon Press: Oxford, UK, 2002.

15. Incurvati, L. On The Consequences of the Definitional Unprovability of Hume's Principle. In Contemporary Perspectives on Logicism and the Foundations of Mathematics; Joray, P., Ed.; Université de Neuchâtel: Neuchâtel, Switzerland, 2007.

16. Boolos, G. Is Hume's Principle analytic? In Language, Thought, and Logic; Oxford University Press: Oxford, UK, 1997; pp. 245-261.

17. Joray, P. A New Path to the Logicist Construction of Numbers. Travaux de logique 2007, 18, 147-165.

18. Gessler, N. Abstraction and Nominalization in Lesniewski's Ontology. Travaux de logique 2007, 18, 63-82.

19. Urbaniak, R. Słupecki's Generalized Mereology and Its Flaws. Hist. Philos. Log. 2014, 35 289-300.

20. Joray, P. Logicism in Leniewski's Ontology. Log. Trianguli 2002, 6, 3-20.

21. Urbaniak, R. Plural Quantifiers. A modal interpretation. Synthese 2013, 191, 1-22.

22. Urbaniak, R. Numbers and Propositions Versus Nominalists: Yellow Cards for Salmon \& Soames. Erkenntnis 2012, 77, 381-397.

23. Simons, P. Lesniewski and Ontological Commitment. In Stanislaw Lesniewski Aujourd'hui; Miéville, D.; Vernant, D., Eds.; Number 16 in Recherches Philosophie, Langages et Cognition, Université de Grenoble: Grenoble, France, 1995; pp. 103-119.

24. Simons, P. Higher-Order Quantification and Ontological Commitment. Dialectica 1997, 51, 255-271.

25. Shapiro, S. Modality and Ontology. Mind 1993, 102, 455-481.

26. Czarnecki, M. Foundations of Mathematics without Actual Infinity. Ph.D. Thesis. University of Warsaw, Warsaw, Poland, 2014.

27. Mostowski, M. On representing concepts in finite models. Math. Log. Q. 2001, 47, 513-523.

28. Czarnecki, M.; Godziszewski, M.T.; Kalociński, D. Language, Life, Limits. In Proceedings of the 10th Conference on Computability in Europe (CiE 2014), Budapest, Hungary, 23-27 June 2014; Springer International Publishing: Cham, Switzerland, 2014; pp. 113-122.

29. Srzednicki, J.; Stachniak, Z. S. Leśniewski's Lecture Notes in Logic; Kluwer Academic Publishers: Dordrecht, The Netherlands, 1988.

(C) 2016 by the author; licensee MDPI, Basel, Switzerland. This article is an open access article distributed under the terms and conditions of the Creative Commons Attribution (CC-BY) license (http://creativecommons.org/licenses/by/4.0/). 